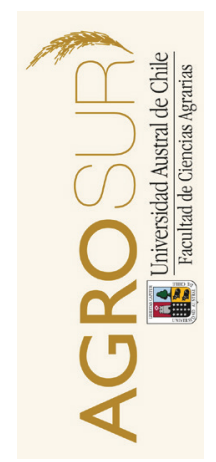

\title{
Relación entre la temperatura timpánica y las condiciones climáticas invernales en novillos en engorda en la Región del Biobío
}

\author{
Relationship between tympanic temperature and climatic variables \\ in steers finished in Biobío region during the wintertime \\ Arias, R.A. ${ }^{* 1}$, Velásquez, A.C. ${ }^{\Psi \ddagger}$, Keim, J.P. , Gandarillas, M. , Alvarado-Gilis, C.A. ${ }^{*}$ \\ *Instituto de Producción Animal, Universidad Austral de Chile, Valdivia-Chile. \\ ${ }^{\Psi}$ Escuela de Agronomía. \\ ‡Núcleo de Investigación en Producción Agroalimentaria, Universidad Católica de Temuco.
}

\begin{tabular}{l} 
A R T I C L E I N F O \\
\hline Article history: \\
Received 29.06.2016 \\
Accepted 21.10.2016 \\
\hline Keywords: \\
Beef cattle \\
Body temperature \\
Thermal comfort \\
Wintertime \\
\hline Original Research Article, \\
Animal Science \\
\hline *Corresponding author: \\
Rodrigo Arias \\
E-mail address: \\
rodrigo.arias@uach.cl
\end{tabular}

ART ICLE INFO

Article history:

Received 29.06.2016

Keywords:

Beef cattle

Body temperature

Thermal comfort

Wintertime

Original Research Article,

Animal Science

Rodrigo Arias

rodrigo.arias@uach.cl

\begin{abstract}
A B S T R A C T
A total of 20 crossbreed steers received an electronic device in the tympanic canal to collect tympanic temperatures (TT). Simultaneously, climatic variables including air temperature, wind speed, solar radiation, relative humidity, and rainfall were collected by using a weather station located at the feedyard, and used to estimate the comprehensive climate index (CCI). Statistical analysis of data included descriptive, ANOVA, simple and multiple lineal regressions with a level of significance of $5 \%$. Climate variables and the CCI were the predictors variables, whereas TT was the response variable. Mean TT of steers was $37.59 \pm 0.02$ and $37.16 \pm 0.03^{\circ} \mathrm{C}$ for years 2010 and 2011, respectively. Climatic conditions during the collecting periods were considered as normal and not challenging for the animals from a thermal balance standpoint. The previous is reflected in the mean values of the CCI, $7.30 \pm 0.11$ and $6.27 \pm 0.12{ }^{\circ} \mathrm{C}$ for years 2010 and 2011 , respectively. Linear regression analyses showed that most of the observed variables were significant but none of them explains in good shape the observed variability $\left(r^{2}<0.10\right)$. Multiple regression analyses improved the value of the coefficients of determination $\left(\mathrm{R}^{2}\right)$, but they still were lower than 0.50 . Finally, we conclude that for the periods of study, TT did not undergo major changes due to environmental conditions of factors assessed. Similarly, the steers showed no signs of environmental stress. Nevertheless, days of data collection were not representative of uncomfortable winter conditions.
\end{abstract}

\section{RESUMEN}

Un total de 20 novillos recibieron un dispositivo electrónico para colectar temperaturas timpánicas (TT). Simultáneamente, también se colectaron las variables climáticas incluyendo: temperatura del aire, velocidad del viento, radiación solar, humedad relativa y precipitación, mediante una estación meteorológica situada en el mismo predio. Estos datos se utilizaron para estimar el índice integral del clima (CCI). El análisis estadístico de los datos incluyó análisis descriptivos, ANDEVA y regresiones simples y múltiples (nivel de significancia del 5\%), siendo TT la variable respuesta. La TT promedio de novillos fue de 37,59 $\pm 0,02$ y 37,16 $\pm 0,03{ }^{\circ} \mathrm{C}$ durante los años 2010 y 2011, respectivamente. Las condiciones climáticas fueron normales y no representaron un desafío para los animales desde el punto de vista térmico. Lo anterior se vio reflejado en los valores medios del CCI de 7,30 $\pm 0,11$ y $6,27 \pm 0,12^{\circ} \mathrm{C}$, durante los años 2010 y 2011, respectivamente. Los análisis de regresión lineal mostraron que la mayoría de las variables observadas fueron significativas, pero ninguna de ellas explica en buena forma la variabilidad observada $\left(r^{2}<0,1\right)$. Los análisis de regresión múltiple mejoraron los valores de los coeficientes de determinación $\left(\mathrm{R}^{2}\right)$, pero aún estuvieron por debajo de 0.50. Finalmente, se concluye que para los períodos de estudio, la TT no sufrió grandes cambios como consecuencia de las condiciones ambientales evaluadas. Del mismo modo, los novillos no mostraron signos de estrés ambiental.

Palabras clave: ganado bovino de engorda, temperatura corporal, confort térmico, invierno.

\section{INTRODUCCIÓN}

La investigación del impacto del cambio climático sobre las respuestas fisiológicas y productivas de los animales representan un desafío (Sprott et al., 2001). En Chile es un tema incipiente que está adquiriendo mayor relevancia, particularmente por la importancia que ha adquirido el tema del bienestar animal. A la fecha, diversas investigaciones han demostrado que tanto el rendimiento como la salud y el bienestar del ganado están siendo marcadamente afectados por las condiciones climáticas (Mader, 2003; Brown-Brandl et al., 2005; Brown-Brandl et al., 2006). En efecto, el adecuado funcionamiento del organismo es el resultado de procesos químicos y físicos que son sensibles a los cambios del medio ambiente circundante. No obstan- 
te lo anterior, los bovinos suelen ser muy adaptables a distintas condiciones ambientales, siendo capaces de lograr un buen desempeño productivo en una amplia gama de entornos. Para ello activan distintos mecanismos fisiológicos y de comportamiento con el objeto de mantener la temperatura corporal (TC) dentro de un rango normal (Sacristán, 1987; Arias et al., 2008). Lo anterior, a su vez, permite a los animales alcanzar la homeostasis necesaria para lograr un adecuado funcionamiento del hipotálamo, el que a su vez tiene un papel vital en la regulación endocrina y en las funciones inmunológicas. Además, el hipotálamo también tiene un rol central en la regulación del consumo de alimento (Cossins y Bowler, 1987), y en consecuencia en la respuesta productiva de los animales. Para lograr mantener la TC dentro de un ajustado margen, el animal debe ganar o perder calor del medio ambiente circundante (balance térmico), proceso que resulta ser muy dinámico y complejo (Bianca, 1968; Hahn et al., 1993).

La TC es afectada por diversos factores, algunos incrementándola tales como la edad, actividad física, alimentación, el celo y la última etapa de la gestación; o bien disminuyéndola como es el caso de la desnutrición, esquilado, e ingestión de grandes cantidades de agua (Bianca, 1976). La TC normal del ganado bovino adulto sano fluctúa entre 37,8 y $40,0{ }^{\circ} \mathrm{C}$ (FAO, 1995), pero está sujeta a modificaciones menores dentro del día ajustándose a un ritmo circadiano (da Silva y Minomo, 1995; Mader y Johnson, 2010; Arias et al., 2011). Dentro de este rango, las actividades celulares y bioquímicas operan con mayor eficiencia y eficacia, pero si los tejidos se enfrían demasiado el metabolismo es entonces reducido. En el caso contrario el metabolismo se acelera, existiendo también el riesgo de desnaturalización de las proteínas, disrupción de la integridad de la membrana celular y posiblemente un daño permanente de los tejidos (Guyton y Hall, 2006), resultando finalmente en morbilidad de largo plazo y bajo desempeño productivo. Entonces, cuando los animales se encuentran dentro de la denominada zona de termoneutralidad, la energía de la dieta es utilizada con mayor eficiencia para mantención, crecimiento, producción y actividad física. En tanto que, cuando las condiciones ambientales se escapan de esta zona la energía es reorientada a funciones tendientes a mantener la condición homeotérmica; y en algunos casos puede existir un aumento en la demanda de energía para estos procesos (Collin et al., 2001; Mader, 2011).

El estudio conjunto del efecto de diversos factores sobre el balance térmico de los animales (radiación solar, humedad relativa, temperatura ambiental, velocidad del viento, precipitaciones, tipo de dieta, nivel energético de la dieta, genotipo, etc.) ha generado un importante conocimiento sobre cómo los animales reaccionan frente a particulares combinaciones de factores climáticos y de manejo productivo (Finch, 1986;
Hahn et al., 2003; West, 2003). En conjunto, estas variables tienen un efecto directo sobre el bienestar animal, así como también en los índices productivos y reproductivos. Entre los elementos del clima que son de importancia directa en la adaptación animal a eventos climáticos de olas de calor y al frío se encuentran: temperatura ambiente, humedad relativa, radiación solar y la velocidad del viento (Arias y Mader, 2009; Hahn et al., 1993). Existen también otros factores indirectos tales como: pluviometría, luz, nubosidad y presión atmosférica (Hahn et al., 2003).

Por otra parte, la temperatura timpánica (TT) sirve como un referente confiable de la temperatura corporal y del bienestar térmico de un animal (Benzinger, 1959; Benzinger, 1964; Easton et al., 2007), por lo que el presente estudio utiliza la medición de la TT en novillos de engorda como una importante variable de confort térmico. Sobre la base de expuesto, el presente estudio tuvo por objetivo general evaluar la relación de la TT de novillos en engorda bajo condiciones comerciales con las variables climáticas del periodo invernal en la Región del Biobío. En tanto los objetivos específicos fueron: a) caracterizar el patrón diario de TT del ganado en condiciones de engorda a corral comercial, y b) establecer relaciones entre los valores de TT, los factores climáticos y el índice integral del clima.

\section{MATERIALES Y MÉTODOS}

El estudio se efectuó en el Fundo Laguna Verde, ubicado aproximadamente a $20 \mathrm{~km}$ al norte de la ciudad de Los Ángeles, Región del Biobío durante los años 2010 y 2011. En cada año se utilizaron 10 novillos híbridos (Angus x Wagyu), de aproximadamente 14 a 15 meses de edad, los cuales fueron seleccionados aleatoriamente desde un corral abierto de 250 animales con aproximadamente $36 \mathrm{~m}^{2}$ /animal, los que disponían de comederos techados y agua en bebederos a libre disposición. Los animales fueron ingresados a una manga siendo inmovilizados mediante un cepo para el procedimiento de instalación y remoción de los dipositivos iButton. Cada uno recibió un dispositivo datalogger del tipo iButton (Maxim Integrated Products Inc., San José, California, Estados Unidos) para colectar la TT. Los dispositivos fueron localizados en el canal timpánico de los animales manualmente y programados para registrar datos a intervalos de 10 minutos. El canal timpánico fue sellado con material absorbente (Tampones Palitex Edgewell, Santiago, Chile) y posteriormente se utilizó un tubo de aislación de espuma (Isoplast, Santiago Chile), para otorgar firmeza a la estructura del pabellón auricular, y así envolver esta con venda elástica (Coban Nexcare 3M, Santiago Chile). Finalmente, se selló con una cinta autoadhesiva de 4 centímetros de ancho (Leukoplast BSN Medical, México DF, México). Una vez finalizado el procedimiento de instalación los 
animales fueron devueltos al corral junto a los otros animales, en donde permanecieron por un período de 9 días, luego de los cuales los dispositivos fueron removidos manualmente. Los animales se encontraban en una condición corporal entre 3 y 4,5 (escala de 1 a 9) y se encontraban recibiendo una dieta ad libitum.

En forma simultánea se colectaron también los datos climáticos mediante una estación meteorológica ubicada aproximadamente a $1 \mathrm{~km}$ al norte del lugar en que se encontraban los corrales de engorda. Las variables observadas correspondieron a: velocidad del viento $(\mathrm{m} / \mathrm{s})$, humedad relativa $(\%)$, temperatura ambiente $\left({ }^{\circ} \mathrm{C}\right)$, radiación solar $\left(\mathrm{W} / \mathrm{m}^{2}\right)$ y precipitación $(\mathrm{mm} /$ día $)$, todas ellas a intervalos de 15 minutos. Posteriormente, tanto los valores de TT como los de las variables climáticas fueron promediados a intervalos de una hora para su posterior análisis.

\section{Análisis de los datos}

La información colectada fue analizada mediante el paquete estadístico JMP 11.0 (SAS Institute, Cary, Carolina del Norte, Estados Unidos). Los datos de TT y de clima fueron procesados y analizados para obtener valores descriptivos y se utilizó un ANOVA (5\% significancia) para comparar los valores entre años. Posteriormente, se realizaron pruebas de regresión simple y múltiple para cada año y también agrupando todos los datos para obtener modelos de predicción de la TT en función de los factores climáticos o del índice íntegral del clima (CCI), este último un indicador de confort térmico para los animales. El CCI se estimó a partir de los registros climáticos utilizando la siguientes ecuación $\mathrm{CCI}=\mathrm{TA}+\mathrm{E} 1+\mathrm{E} 2+\mathrm{E} 3$ (Mader et al., 2010). Donde, E1 a E3 corresponden a factores de corrección de las temperatura ambiente estimados a partir de:

\section{RESULTADOS Y DISCUSIÓN}

El Cuadro 1 muestra el resumen de las variables climáticas colectadas en el estudio por día y año, así como también del CCI. Se observa que para el periodo de colecta de datos la TA, en ambos años, estuvo por sobre $0{ }^{\circ} \mathrm{C}$, siendo el promedio del 2011 superior al del 2010 $(\mathrm{P}<0,001)$. El menor valor de CCI en el año 2010 se explica por la mayor VV $(\mathrm{P}<0,001)$ y menor RS $(\mathrm{P}<0,001)$ en comparación con la semana de colecta de datos del 2011. En el caso de las precipitaciones, se registraron solo dos días con lluvia durante el 2010, concentrándose una alta pluviometría en el último día de colecta de datos. En tanto, en el segundo periodo (2011), las precipitaciones fueron homogéneas durante todos los días de colecta de datos. Cabe señalar que en el 2010 se observó una disminución en la TT asociada a los eventos de precipitaciones y, a su vez, que en esos mismos días o bien el día previo, se registró un aumento en la velocidad del viento.

La información obtenida en el presente estudio, muestra diferencias entre los valores promedios de CCI vs. TA en cada periodo $\left(2,15\right.$ vs. $\left.3,97^{\circ} \mathrm{C}\right)$, las que se explican mayormente por el efecto del viento y en menor medida por los cambios en la radiación solar. En este caso el CCI representa la sensación térmica que el animal percibe al ser ser corregida por HR, VV y RS. No obstante las diferencias, estos valores se encuentran dentro de la zona termoneutral para animales en engorda.

La Figura 1 muestra la relación de la TT de los novillos con el CCI durante los días de las semanas evaluadas. En el 2010 los valores de TT presentaron menor variación entre días en comparación con el 2011. Se aprecia que en el 2010 los valores promedio de CCI disminuyeron al avanzar los días, pero los valores de TT en general aumentaron, con la excepción de los días en

Ecuación 1: Factor de corrección por temperatura y humedad:

$$
e^{\left(0,00182 * H R+1,8 * 10^{-5} * T A * H R\right)} *\left(0,000054 * T A^{2}+0,00192 * T A-0,0246\right) *(H R-30)
$$

Ecuación 2: Factor de corrección por velocidad del viento:

$$
\left[\frac{-6,56}{e^{\left\{\left(\frac{1}{(2,26 \times V V+0,23)^{0,45}}\right) \times\left(2,9+1,14 \times 10^{-6} \times V V^{2,5}-\log _{0,3}(2,26 \times V V+0,33)^{-2}\right)\right\}}}\right]-0,00566 \times V V^{2}+3,33
$$

Ecuación 3: Factor de corrección por radiación total:

$$
0,0076 * R S-0,00002 * R S * T A+0,00005 * T A^{2} * \sqrt{R S}+0,1 * T A-2
$$

Donde $\mathrm{TA}=$ temperatura ambiental $\left({ }^{\circ} \mathrm{C}\right)$; $\mathrm{HR}=$ humedad relativa $(\%)$; $\mathrm{VV}=$ velocidad del viento $(\mathrm{m} / \mathrm{s})$; $\mathrm{RS}=$ radiación solar $\left(\mathrm{W} / \mathrm{m}^{2}\right)$. La base de datos fue depurada considerando sólo aquellos días en que se contó con registros completos TT. 


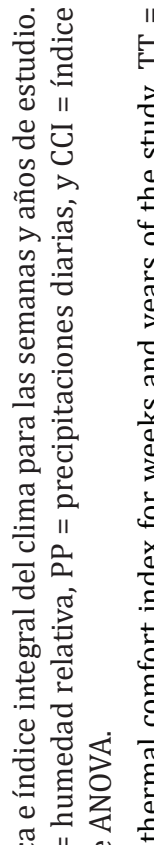

đI 11 \&

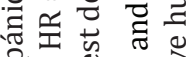

छㅊ:

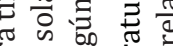

吨

范言

: 0 .

ఖँ

đֶ?

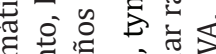

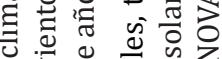

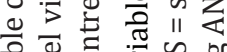

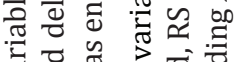

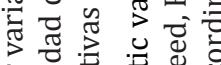

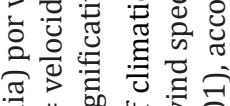

范 11 .

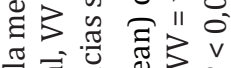

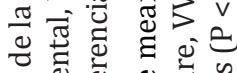

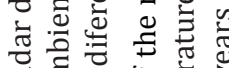

䒕考

๒ँ

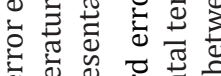

+

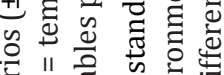

可的䨌

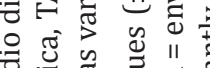

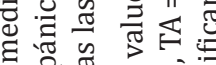

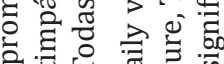

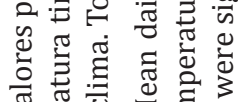

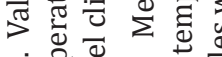

-

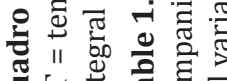

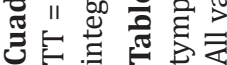

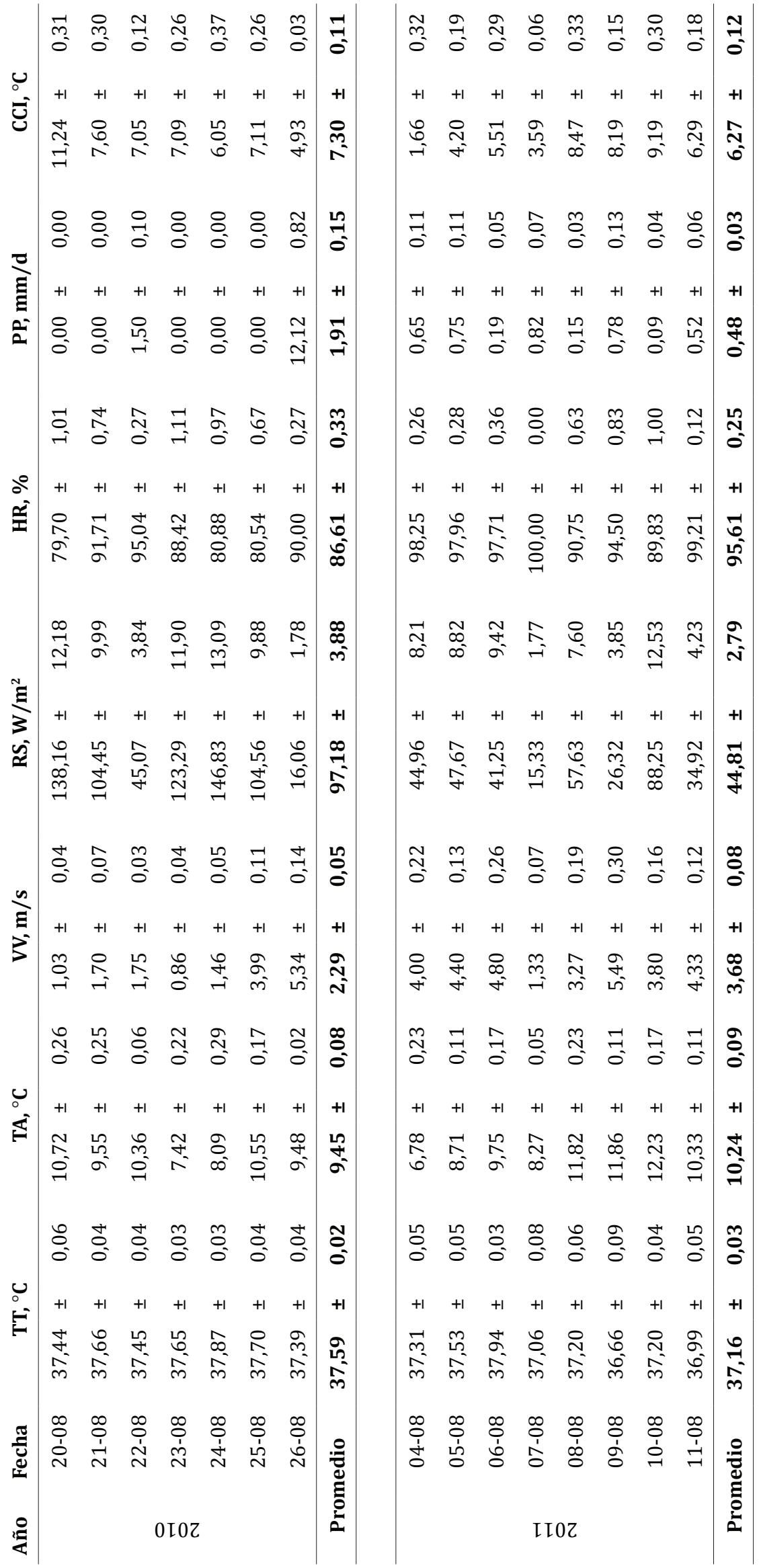




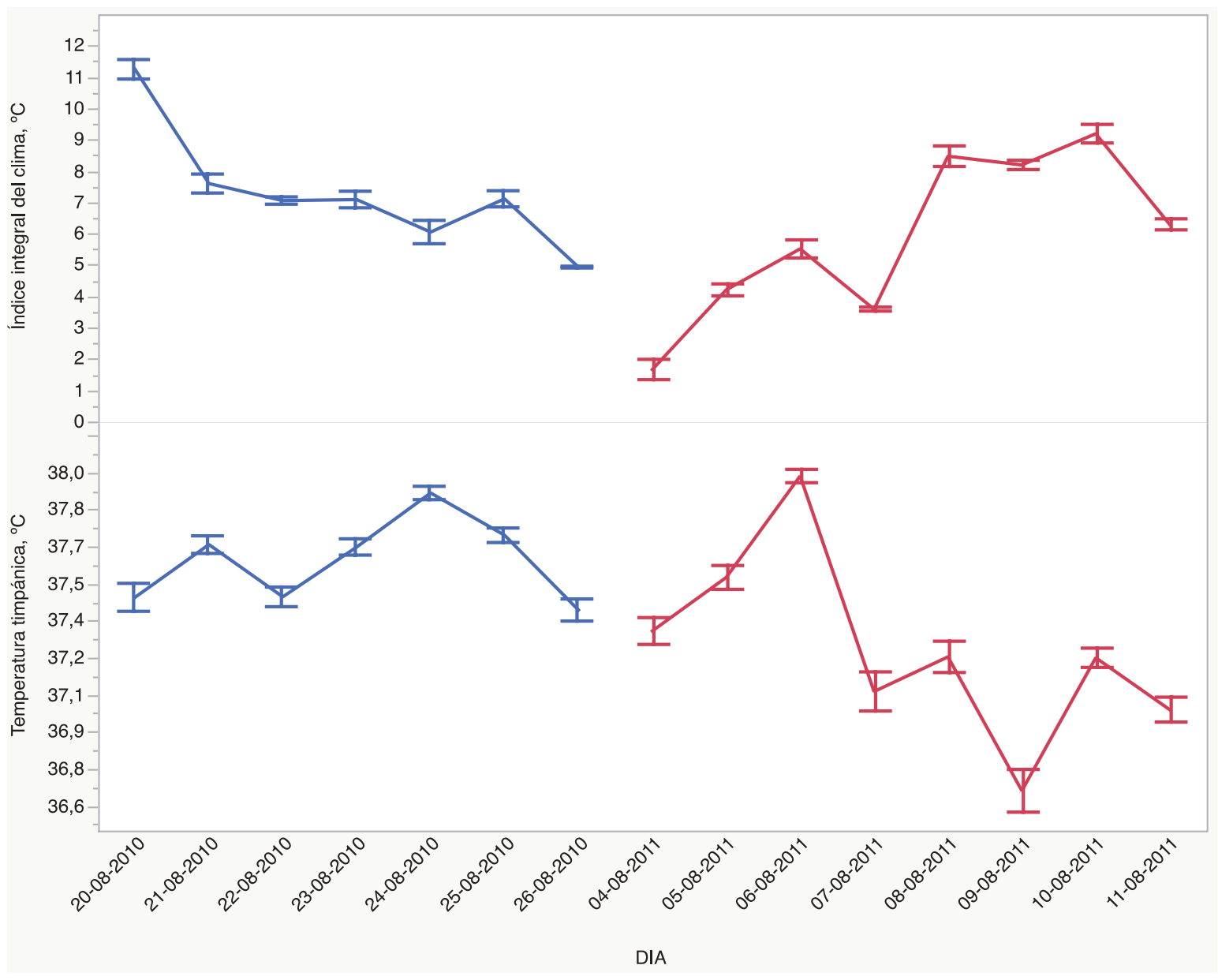

Figura 1. Valores promedios diarios ( \pm error estándar de la media) de temperatura timpánica y del índice integral del clima para los periodos evaluados.

Figure 1. Mean daily values ( \pm standard error of the mean) of tympanic temperature and comprehensive climatic index for the evaluated periods.

que se registraron precipitaciones (22 y 25 de agosto 2010). En el 2011 se observó un patrón muy similar entre CCI y TT, con las mayores bajas en TT asociadas a los eventos de días con mayores precipitaciones $(7$ y 9 de agosto 2011). Cabe señalar que el algoritmo para estimar CCI no incluye las precipitaciones, pero éstas afectan el balance térmico del animal al formar barro lo que cambia la demanda de energía de mantención en los animales (Bond et al., 1970).

La Figura 2 muestra la relación entre la TT y hora del día, según periodo, promediando los valores de todos los días y animales. Se aprecia un patrón similar en el CCI, el cual aumentó después de las 07:00 h, lo que coincide con el incremento de la RS y TA. Los valores máximos de CCI se observaron a las 14:00 h. No obstante, los valores de CCI fueron distintos entre los años $(\mathrm{P}<0,001)$. En tanto los valores de TT presentaron un patrón similar, pero con valores distintos a través del día $(\mathrm{P}<0,001)$ entre años. La TT más baja se registró a las 09:00 h y la más alta alrededor de las 20:00 a 21:00 $\mathrm{h}$, existiendo una diferencia de 1,05 y de $0,89{ }^{\circ} \mathrm{C}$ entre los valores máximos y mínimos de los años 2010 y 2011, respectivamente.

En la actualidad existen diferentes metodologías de registro de temperatura utilizadas en rumiantes, presentando cada una de ellas limitaciones y ventajas. Por ejemplo, Lowe et al. (2001), indicaron que los registros de TT son problemáticos si es que los termopares se llegan a desprender, resultando una pérdida de datos. En el caso de este ensayo, no se utilizaron termopares, sino dispositivos que no requieren de conexión alguna, previniendo este problema. Por su parte, Bluett et al. (2000) demostraron con éxito la aplicación intravaginal de dispositivos para medir la temperatura corporal en vacas lecheras. Sin embargo, los dispositivos son claramente sólo aplicables a hembras. También existen registros de implantes quirúrgicos abdominales para el registro de temperatura corporal (Kamerman et al., 


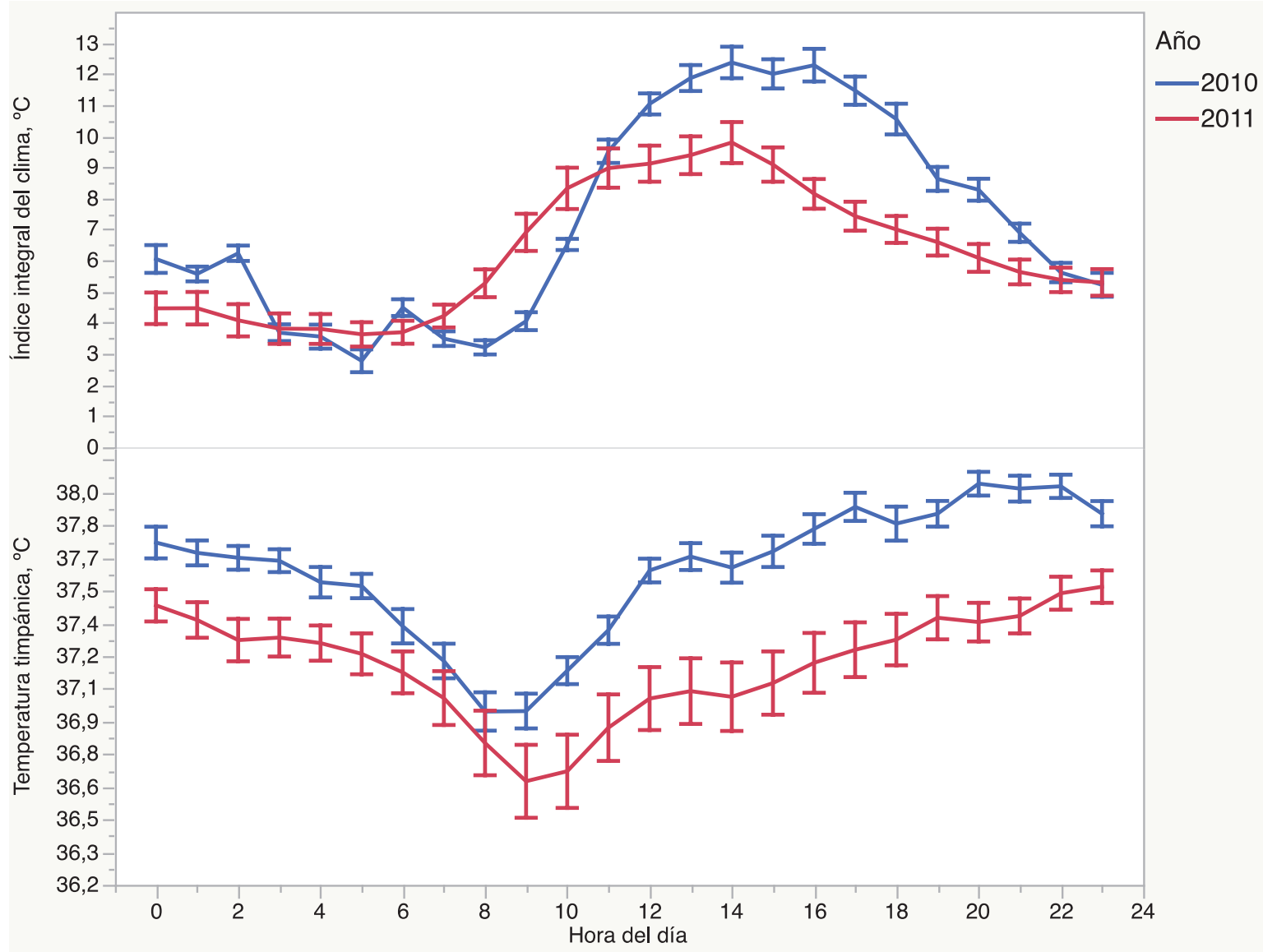

Figura 2. Valores promedios por hora del día ( \pm error estándar de la media) de temperatura timpánica y del índice integral del clima para los periodos evaluados.

Figure 2. Mean hourly values ( \pm standard error of the mean) of tympanic temperature and comprehensive climatic index for the evaluated periods.

2001), procedimiento consistente en una cirugía invasiva con el riesgo de infecciones. Tambien se encuentran aquellos que se ingieren y permanecen en el rumen (Eigenberg et al., 2008). Éstos últimos ofrecen una medición precisa y continua pero son más costosos, no se pueden recuperar y se utilizan solo con fines experimentales. Otra alternativa la constituyen los dispositivos intrarrectales, los que han sido exitosamente utilizados (Gaughan et al., 1999). Sin embargo, requiere un registro con un dispositivo externo, lo que significa que el ganado debe ser alojado en corrales donde se les restrinja el normal movimiento y comportamiento. Algunas de estas alternativas podrían ser cuestionados al afectar el bienestar animal.

La utilización de los dispositivos de tipo iButton permitió contar con registros continuos de TT, monitoreando de mejor manera la respuesta animal en términos energéticos en comparación a la temperatura rectal que solo muestra un momento en particular. Estos dispositivos son más prácticos debido a su facilidad de instalación y a las mínimas molestias que le confieren al animal (Guidry y McDowell, 1966). Benzinger (1964), por otra parte, concluyó que aunque la medición de la temperatura en la membrana timpánica no es tan rápida como otras medidas de la temperatura intracraneal, exhibe los mismos patrones esenciales después de la estimulación externa e interna y, por lo tanto, se puede utilizar como una medida de temperatura intracraneal bajo estrés térmico de uso a corto plazo (Guidry y McDowell, 1966). Lo anterior nos permite tener confianza en los registros obtenidos en el presente estudio.

\section{Modelos de regresión simple y múltiple}

El Cuadro 2 resume los valores de los coeficientes de determinación y sus respectivas significancias para cada una de las variables climáticas y de CCI utilizadas como predictores de TT. Si bien prácticamente todas las variables observadas son significativas en cuanto a su efecto en la TT, con la excepción de VV en el 2011 y de las PP en el modelo global (combinando ambos años), ninguna de ellas explica de buena forma la va- 
riabilidad observada, dado que los valores de los coeficientes de determinación ajustado $\left(\mathrm{r}^{2}\right)$ fueron todos inferiores a 0,50 .

El Cuadro 3 muestra las ecuaciones obtenidas del análisis de regresión múltiple $(\mathrm{P}<0,001)$, las que solo incluyeron variables climáticas como predictoras de la TT. No se incluyó en este caso al CCI, dada la colinealidad existente con las variables climáticas, pues el CCI las incluye en su estimación. Se observó un incremento en los valores de los coeficientes de determinación $\left(\mathrm{R}^{2}\right)$ en comparación con los análisis de regresión lineal simple, pero aún siendo estos bastante bajos $(<0,5)$. Lo anterior indicaría que existen otros factores además de los climáticos que están incidiendo en los valores de TT observados.

Si bien el periodo de colecta de datos fue limitado a unos pocos días en cada año, la presencia de días con lluvias se asoció negativamente con la TT de los animales, especialmente en el año 2010. Cabe señalar que hasta hace unos pocos años la mayoría de los estudios de balance térmico se realizaban en condiciones con- troladas con la imposibilidad de recrear las condiciones productivas en las que los animales crecen y se reproducen (Arias et al., 2008). Sin embargo, los avances tecnológicos han permitido contar con dispositivos electrónicos y telemétricos para registrar cambios de TT y en la tasa de respiración (entre otros), lo que ha facultado el desarrollo de investigaciones de campo o bajo condiciones comerciales. Con ello, se han logrado avances significativos en el entendimiento de cómo reaccionan los animales a factores climáticos (Arias et al., 2008). Así, aun cuando la TA es la variable probablemente más investigada y al mismo tiempo la más utilizada como indicador de estrés en el ganado (Hahn et al., 2003), por sí sola no es una adecuada expresión de la respuesta animal al estrés térmico (Khalifa, 2003), ya que la sensación térmica a la que se expone el ganado puede ser alterada por la acción del viento, humedad, precipitación y radiación entre otros factores (NRC, 1981).

Estudios realizados en el oeste de Canadá han demostrado que durante el período invernal, las variables

Cuadro 2. Coeficientes determinación de regresión lineal simple para variables climáticas y sus respectivos valores de significancia.

Table 2. Coefficients of determination of simple linear regression of climatic variables and levels of significance.

\begin{tabular}{lcrrrrr}
\hline \multirow{2}{*}{\multicolumn{1}{c}{ Variable }} & \multicolumn{2}{c}{$\mathbf{2 0 1 0}$} & \multicolumn{2}{c}{$\mathbf{2 0 1 1}$} & \multicolumn{2}{c}{ Ambos años } \\
\cline { 2 - 7 } & \multicolumn{1}{c}{$\mathbf{r}^{2}$} & P-value & \multicolumn{1}{c}{$\mathbf{r}^{2}$} & P-value & \multicolumn{1}{c}{$\mathbf{r}^{2}$} & P-value \\
\hline Velocidad del viento (VV) & 0,03 & 0,0231 & 0,01 & 0,0530 & 0,02 & $<0,0001$ \\
Temperatura ambiental (TA) & 0,06 & $<0,0001$ & 0,01 & 0,0045 & 0,01 & $<0,0001$ \\
Humedad relativa (HR) & 0,01 & $<0,0001$ & 0,01 & 0,0367 & 0,08 & $<0,0001$ \\
Radiación solar (RS) & 0,01 & 0,0014 & 0,02 & $<0,0001$ & 0,01 & 0,0003 \\
Precipitaciones (PP) & 0,01 & 0,0005 & 0,02 & $<0,0001$ & 0,00 & 0,1241 \\
Índice integral del clima (CCI) & 0,04 & $<0,0001$ & 0,01 & 0,0004 & 0,02 & $<0,0001$ \\
\hline
\end{tabular}

Cuadro 3. Valores de los coeficientes de los parámetros y del coeficiente de determinación del análisis de regresión múltiple para A) ecuación año 2010, B) ecuación año 2011, y C) ecuación combinando ambos años.

Table 3. Coefficients for parameters of the multiple regressions and its coefficients of determination for A) year 2010, B) year 2011 and C) overall equation.

\begin{tabular}{llc}
\hline Ecuación & & $\mathrm{R}^{2}$ \\
\hline A) & $\mathrm{TT}=38,8443+0,0315 \mathrm{TA}-0,0573 \mathrm{VV}-0,0009 \mathrm{RS}-0,0154 \mathrm{HR}$ & 0,1201 \\
B) & $\mathrm{TT}=41,1608-0,0856 \mathrm{TA}-0,0015 \mathrm{RS}-0,0313 \mathrm{HR}-0,1357 \mathrm{PP}$ & 0,0922 \\
C) & $\mathrm{TT}=40,0390-0,0665 \mathrm{VV}-0,0011 \mathrm{RS}-0,0259 \mathrm{HR}$ & 0,1451 \\
\hline
\end{tabular}

Todas las ecuaciones significativas $(\mathrm{P}<0,0001)$.

$\mathrm{TT}$ = temperatura timpánica, $\mathrm{TA}=$ temperatura Ambiental, $\mathrm{VV}=$ velocidad del viento, $\mathrm{RS}=$ radiación solar, $\mathrm{HR}=$ humedad relativa, $\mathrm{y}$ PP = precipitaciones diarias. 
climáticas en general, y la TA promedio en particular, afectan fundamentalmente la ganancia diaria de peso y la cantidad de energía (Mcal) requerida por cada kilogramo de peso ganado (Christison y Milligan, 1974). La menor productividad durante el invierno está asociada a mayor demanda de energía para mantención y a menor digestibilidad del alimento. Por su parte Mader (2003), reportó que las ganancias de peso no se ven afectadas entre estaciones del año, salvo en la primavera en que ésta es superior. En climas fríos, Bond et al. (1970) reportaron que el barro reduce la ganancia diaria de peso de los animales en un 25 a $37 \%$ y que incrementa la cantidad de alimento requerido por $\mathrm{kg}$ de peso ganado en un 20 a 33\%. Cabe señalar que en el presente estudio no fue posible contar con información de consumo ni ganancias de peso como para poder corroborar lo antes expuesto. Un aspecto importate a considerar es que si bien los inviernos en el sur de Chile (zona en que se colectaron los datos) son menos extremos que los del medio oeste de Estados Unidos y Canadá en términos de temperatura ambiental y presencia de nieve, si presentan periodos más extensos con barro en los corrales en Chile (abril a noviembre), mientras que en el hemisferio norte las precipitaciones líquidas ocurren en el periodo primavera-verano.

No obstante lo anterior, es posible señalar que las condiciones ambientales de los periodos invernales evaluados no representaron un desafío térmico para los animales, pues no se observaron signos evidentes de estrés, salvo la acumulación de barro. Esto último a pesar de que el diseño de los corrales contaba con suficiente espacio y con montículos, siguiendo las recomendaciones de Estados Unidos para corrales de engorda.

\section{CONCLUSIÓN}

El estudio realizado permitió caracterizar la evolución de la temperatura timpánica en novillos de 14 a 15 meses de edad en engorda a corral bajo condiciones comerciales. Asimismo, en el periodo de estudio no se detectaron alteraciones que pudieran indicar estrés térmico basándose en el índice integral del clima. Se pudo establecer que las temperaturas, tanto timpánica como ambiental, la velocidad del viento, la radiación solar y los índices de confort animal mostraron una correlación entre sí, pero no fue posible predecir la temperatura timpánica en base a estas variables climáticas.

\section{Agradecimientos}

Los autores agradecen el trabajo de terreno realizado por los estudiantes Sr. Juan Guzmán A. y al Sr. Pablo Vergara F. Asimismo, agradecen a Chilebeef (Fundo Laguna verde y su personal) por colaboración y ayuda en la realización del presente estudio.

\section{REFERENCIAS}

Arias, R.A., Mader, T.L., Escobar, P.C., 2008. Climatic factors affecting cattle performance in dairy and beef farms. Archivos de Medicina Veterinaria 40(1), 7-22.

Arias, R.A., Mader, T.L., 2009. Effects on environmental factors on body temperature of feedlot cattle, Nebraska Beef Report (Vol. MP92, pp. 102-104.). Lincoln Nebraska: University of Nebraska-Lincoln.

Arias, R.A., Mader, T.L., Parkhurst, A.M., 2011. Effects of diet type and metabolizable energy intake on tympanic temperature of steers fed during summer and winter seasons. Journal of Animal Science 89(5), 1574-1580.

Benzinger, T.H., 1959. On physical heat regulation and the sense of temperature in man. Proceedings of the National Academy of Sciences of the United States of America 45(4), 645-659.

Benzinger, T.H., 1964. The Thermal Homeostasis of Man. Symposium Society for Experimental Biology 18, 49-61.

Bianca, W., 1968. Thermoregulation, in: Hafez E.S.E. (Ed.). Adaptation of Domestic Animals Philadelphia: Lea \& Febiger Publisher, pp. 97-118.

Bianca, W., 1976. The Significance of Meteorology in Animal Production. International Journal of Biometeorology 20(2), 139-156.

Bluett, S.J., Fisher, A.D., Waugh C.D., 2000. Heat challenge of dairy cows in the Waikato: a comparison of spring and summer. Proceedings of the New Zealand Society of Animal Production 60, 226-229.

Bond, T.E., Garrett, W.N., Givens, R.L., Morrison, S.R., 1970. Comparative effects of mud, wind and rain in beef cattle performance. International Journal of Farming. Building Research 5, 3-9.

Brown-Brandl, T.M., Eigenberg, R.A., Hahn, G.L., Nienaber, J.A., Mader, T.L., Spiers, D.E., Parkhurst, A.M., 2005. Analyses of thermoregulatory responses of feeder cattle exposed to simulated heat waves. International Journal of Biometeorology 49(5), 285-296.

Brown-Brandl, T.M., Eigenberg, R.A., Nienaber, J.A., 2006. Heat stress risk factors of feedlot heifers. Livestock Science 105, 57-68.

Christison G.I., Milligan, J.D., 1974. A seven year study of winter performance of feedlot steers in western Canada. Proceeding of the International Livestock Environment Symposium, University of Nebraska-Lincoln, USA, April, 17-19 1974, pp. 296-300.

Collin, A., van Milgen, J., Dubois, S., Noblet, J., 2001. Effect of high temperature on feeding behaviour and heat production in group-housed young pigs. British Journal of Nutrition 86(01), 63-70.

Cossins, A.R., Bowler, K., 1987. Temperature Biology of Animals. Chapman and Hall, London.

da Silva, R.G., Minomo, F.R., 1995. Circadian and seasonal variation of the body temperature of sheep in a tropical environment. International Journal of Biometeorology 39, 69-73.

Easton, C., Fudge, B.W., Pitsiladis, Y.P., 2007. Rectal, telemetry pill and tympanic membrane thermometry during exercise heat stress. Journal of Thermal Biology 32(2), 78-86.

Eigenberg, R.A., Brown-Brandl, T.M., Nienaber, J.A., 2008. Sensors for dynamic physiological measurements. Compu- 
ter and Electronics in Agriculture 62, 41-47.

FA0. 1995. Manual para el personal auxiliar de sanidad animal primaria. http://www.fao.org/docrep/t0690s/ t0690s00.htm (acceso 06.24.2016).

Finch, V.A., 1986. Body temperature in beef cattle: Its control and relevance to production in the tropics. Journal of Animal Science 62(2), 531-542.

Gaughan, J.B., Mader, T.L., Holt, S.M., Josey, M.J., Rowan, K.J., 1999. Heat tolerance of Boran and Tuli crossbred steers. Journal of Animal Science 77(9), 2398-2405.

Guidry, A.J., McDowell, R.E., 1966. Tympanic membrane temperature for indicating rapid changes in body temperature. Journal of Dairy Science 49(1), 74-77.

Guyton, A.C., Hall, J.E., 2006. Textbook of Medical Physiology. Elsevier-Saunders Publishers, Philadelphia, PA.

Hahn, G.L., Nienaber, J.A., Eigenber, R.A., 1993. Environmental influences on the dynamic of thermoregulation and feeding behavior in Cattle and Swine. Proceedings of the IV International Livestock Environment Symposium, University of Warwick, Coventry, England, July, 6-9 1993, pp. 1106-1116.

Hahn, G.L., Mader, T.L., Eigenberg, R.A., 2003. Perspective on development of thermal indices for animal studies and management, in: Lacetera, N., Bernabucci, U., Khalifa, H.H., Ronchi, B., Nardone, A. (Eds.), Interactions between climate and animal production (EAAP Technical Series No. 7), Wageningen Academic Publishers, The Netherlands, pp. 31-44.

Kamerman, P.R., Di Zio, L.C., Fuller, A., 2001. Miniature data loggers for remote measurement of body temperature in medium-sized rodents. Journal of thermal biology 26(3), 159-163.

Khalifa, H.H., 2003. Bioclimatology and adaptation of farm animals in a changing climate, in: Lacetera, N., Bernabucci, U., Khalifa, H.H., Ronchi, B., Nardone, A. (Eds.), Interactions between climate and animal (EAAP Technical Series No. 7), Wageningen Academic Publishers, The Netherlands, pp 15-29.

Lowe, T.E., Cook, C.J., Ingram, J.R., Harris, P.J., 2001. Impact of climate on thermal rhythm in pastoral sheep. Physiology \& Behavior 74, 659-664.

Mader, T.L., 2003. Environmental stress in confined beef cattle. Journal of Animal Science 81(14_suppl_2), E110-119.

Mader, T.L., Johnson, L., 2010. Tympanic temperature profile of confined beef cattle Nebraska Beef Report (Vol. MP93, pp. 101-103.). Lincoln Nebraska: University of Nebraska-Lincoln.

Mader, T.L., Johnson, L.J., Gaughan. J.B., 2010. A comprehensive index for assessing environmental stress in animals. Journal of Animal Science 88(6), 2153-2165.

Mader, T.L., 2011. Mud Effects on Feedlot Cattle Nebraska Beef Report (Vol. MP94, pp. 82-83): University of Nebraska-Lincoln.

MAFF., 2000. Climate change and agriculture in the United Kingdom. Ministry of Agriculture, Fisheries and Food (MAAF). London, UK.

NRC., 1981. Effect of Environment on Nutrient Requirements of Domestic Animals. National Academy Press, Washington DC.

Sacristán, G., 1987. Fisiología veterinaria. Editorial Interamericana/Mc Graw Hill. Madrid.

Sprott, L.R., Selk, G.E., Adams, D.C., 2001. REVIEW: Factors affecting decisions on when to Calve Beef Females. Professional Animal Scientist 17(4), 238-246.

West, J.W., 2003. Effects of heat-stress on production in dairy cattle. Journal of Dairy Science 86, 2131-2144. 
